\title{
Quality of Experience Study for Multiple Sensorial Media Delivery
}

\author{
Zhenhui Yuan, Member, IEEE, Gheorghita Ghinea, Member, IEEE, and Gabriel-Miro Muntean, Member, IEEE
}

\begin{abstract}
Traditional video sequences make use of both visual images and audio tracks which are perceived by human eyes and ears, respectively. In order to present better ultra-reality virtual experience, the comprehensive human sensations (e.g. olfaction, haptic, gustatory, etc) needed to be exploited. In this paper, a multiple sensorial media (mulsemedia) delivery system is introduced to deliver multimedia sequences integrated with multiple media components which engage three or more of human senses such as sight, hearing, olfaction, haptic, gustatory, etc. Three sensorial effects (i.e. haptic, olfaction, and air-flowing) are selected for the purpose of demonstration. Subjective test is conducted to analyze the user perceived quality of experience of the mulsemedia service. It is concluded that the mulsemedia sequences can partly mask the decreased movie quality. Additionally the most preferable sensorial effect is haptic, followed by air-flowing and olfaction.
\end{abstract}

Keywords-QoE, multi-sensory, subjective test.

\section{INTRODUCTION}

$\mathbf{F}$ OR decades on end, traditional movie service takes advantage of both human eyes and ears to provide audiovisual experience. However, the conventional movie sequences have limited the potencies of human senses in their ability to fully initiate the real immersive communication scenarios. For instance, when delivering some movie content, users cannot feel real environmental/ambiental elements such as flavor of the flowers, air motion of the ocean wind, haptic of a push, etc. Recently, three-dimensional (3D) image technology is emerging in the movie industry and has achieved a tremendous business success and ultimately changed the way people enjoying the movie [1]. Meanwhile immersive communication has attracted lots of research attention to present ultra-realistic virtual experience [2]. A new paradigm has been introduced to extend the traditional multimedia sequences with additional components and is referred to as mulsemedia (multiple sensorial media)engaging three or more of human senses [3].

This paper presents a novel mulsemedia delivery system including three sensorial effects (i.e. haptic, olfaction, and airflowing) in order to enhance the traditional movie experience. The system implementation is introduced including the equipment, software and the media sequences used. The system can be extended to provide more human sensorial-media related

This work was supported in part by Enterprise Ireland Innovation Partnership programme.

Z.Yuan and G.-M. Muntean are with the Performance Engineeirng Laboratory, Network Innovations Centre, School of Electronic Engineering (http://www.eeng.dcu.ie/ pel), Dublin City University, Ireland (e-mail: yuanzh, munteang@eeng.dcu.ie).

G. Ghinea is with Brunel University, UK (george.ghinea@brunel.ac.uk). objects such humidity, temperature, etc. Additionlly, extensive subjective tests are conducted to investigate the user perceived quality of experience (QoE) of the mulsemedia service. Results of this paper can be used as deployment recommendations for potential mulsemedia service providers.

This paper is organized as follows. Section II introduces the existing research works related to mulsemedia services. Section III presents the implementation of the mulsemedia delivery system. Section IV shows the subjective tests conducted using the mulsemedia delivery system and analyze the results obtained. Section V concludes the paper.

\section{RELATED WORKS}

Multimedia data, unlike traditional media content that uses text only, refers to a combination of text, still images, animation, audio, and video. Extensive research works have been conducted to deliver multimedia services in IP-based network,a significant fraction of which occurs in real-time, a feature which is generally recognized as multimedia streaming. Advanced solutions have been proposed in [4][5][6]. However, delivery of mulsemedia service is lack of depth investigation.

Olfaction is one of the last challenges which multimedia applications have to conquer. Enhancing such applications with olfactory stimuli has the potential to create a more complex and richer user mulsemedia experience.Pioneering efforts were first carried out by Kaye [7]. His work played a significant role in creating an awareness of the issues, problems and limitations associated with the use of olfactory data, incidentally also serving as a good summary of olfaction incorporation in various applications and industries across the years. Haptic user interfaces have been applied to the domain of human-computer interaction in virtual environments including medical, automotive, mobile phone, entertainment, controls, education, training, rehabilitation, assistive technology, and the scientific study of touch [8].

Incipient efforts in mulsemedia research have been forthcoming. For instance, there have been a few studies carried out to investigate the user-perceived experience associated with the use of the newer media objects such as tactile (touch) and olfactory media objects. However, because the use of these media objects is relatively new in the multimedia field, most of these perceptual studies have concentrated their efforts on the practicality and possibility of incorporating these media objects into these applications. One such research effort is a VR learning system called VIREPSE which provides both olfactory and haptic feedback [9]. In related work, [10] describes an investigative study which explored the possibility of using a vibratory-tactile device on the whole body for 


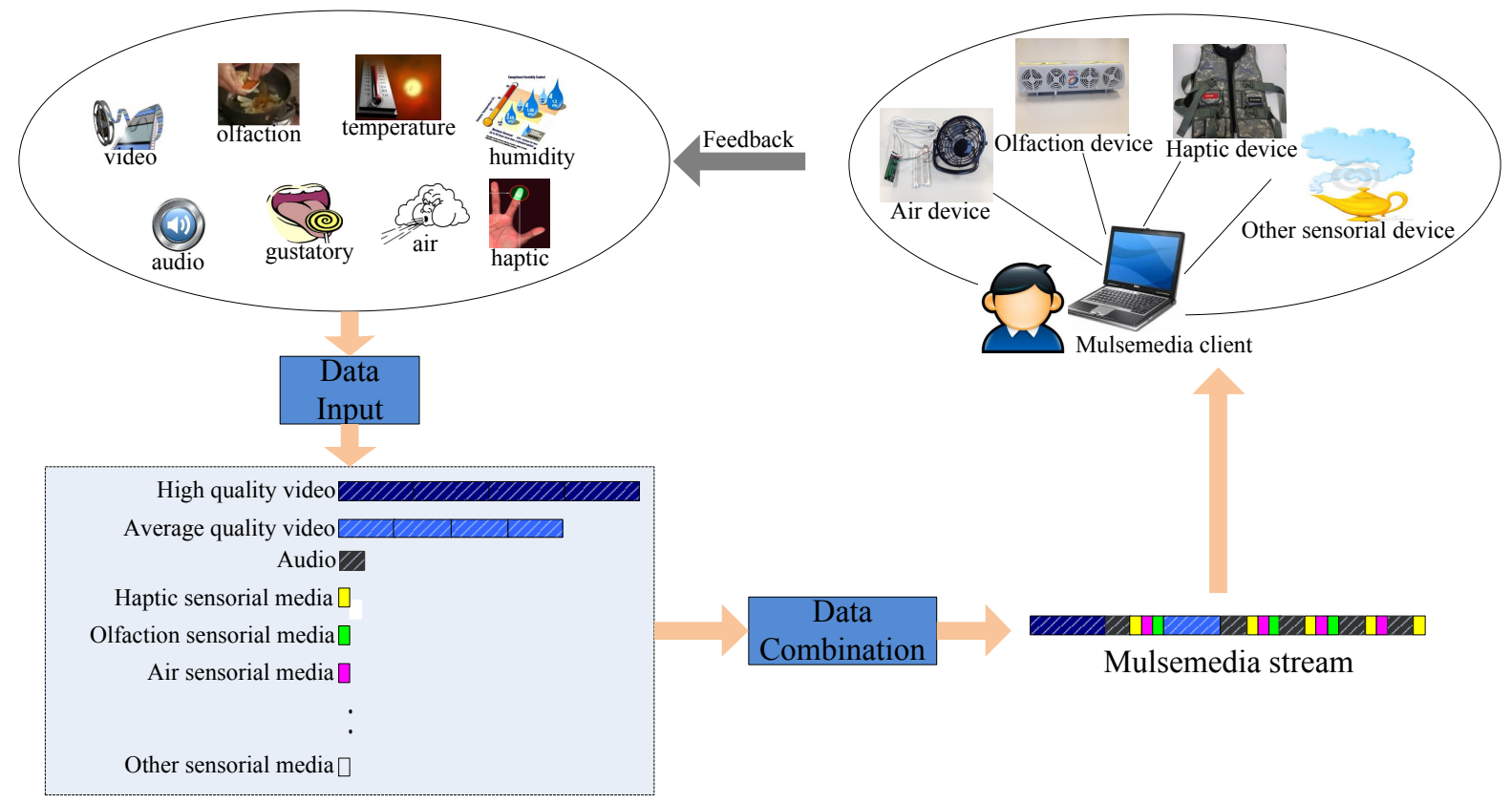

Fig. 1: Overviewed architecture of the mulsemedia delivery system

simulating collision between the user and a VR environment. Results revealed that their proposed vibratory-tactile interface did enhance the sense of presence, especially when combined with 3D sound. In [11] researchers present strategies and algorithms to model context in applications that allow users to haptically explore objects in virtual reality/augmented reality environments. Results show significant improvement in accuracy and efficiency of haptic perception in augmented reality environments when compared to conventional approaches that do not model context in haptic rendering. Indeed, the use of haptic in mulsemedia VR environments has very recently also been the subject of the research reported in [2].

\section{Mulsemedia Delivery System Development}

This section presents the development details of the mulsemedia movie delivery system including architecture of mulsemedia delivery system, mulsemedia sequences selection, and equipment used.

\section{A. Mulsemedia Delivery Architecture}

Figure 1 illustrates a scenario in which the mulsemedia content is delivered to end users. On the left side, the mulsemedia server selects multimedia (i.e. text, video, and audio) and metadata related to a number of sensorial media components. The sensorial media includes olfaction, haptic, air, temperature, humidity, etc. All of these media objects are perceived by the human sensory system (e.g. sight, smell, touch, etc.). Users can inform the mulsemedia server about both network delivery conditions and user preferences and the server then adjusts the multi-sensorial content delivery process accordingly. This paper focuses on the user perceived $\mathrm{QoE}$ of the mulsemedia services rather than the adaptation principle of the mulsemedia data.

\section{B. Mulsemedia Movie Sequences}

Table I shows 32 multimedia clips taken from the two movies, Jurassic Park and Back To The Future. Three sensorial effects (haptic, air-flow and olfaction) were integrated into the 32 multimedia sequences according to the content scenarios as shown in Table I. Each of the two movies includes eight high motion video clips and eight low motion video clips. The individual video clip is 30 seconds long and is manually integrated with certain sensorial effects based on the content. For instance, when the movie plays a scenario of animal attacking (e.g. in Jurassic Park), then the haptic effect is synchronized to provide attacking experience. Each multimedia clip is encoded into two quality levels as shown in Table II. All multimedia sequences have the same codec settings using the MPEG-4.

\section{Mulsemedia Tools}

In order to integrate the multiple sensorial effects into the multimedia sequences, additional equipment and software are required. Figure 2 (a), (b), and (c) show the adopted hardware devices which can provide sensorial effects: a USB fan for air-flowing effect, a scent dispenser for olfaction effect, and a vibration vest for haptic effect. All of the three devices are programmable in order to be synchronized with the multimedia sequences. The USB fan is controlled by a visual basic program using USBmicro 2002 . The scent dispenser (Vortex Active USB) is obtained from Dale Air , who also provides many types of aroma cubes to mimic the actual scent. The scent dispenser comes along with four fans and thus provides 


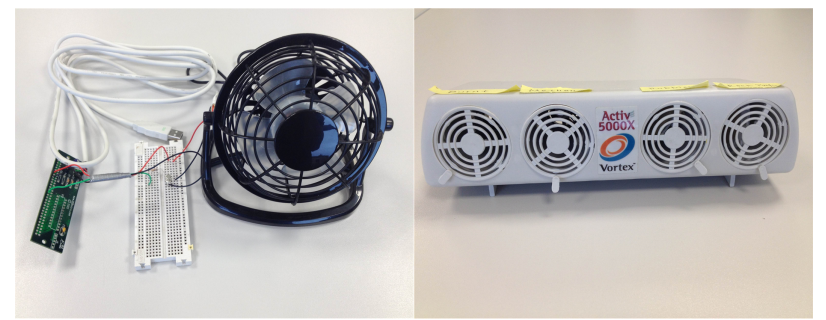

(a) USB fan

(b) Olfaction dispenser

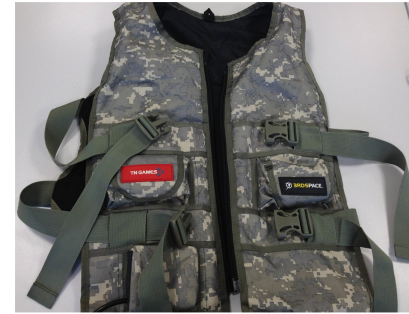

(c) Haptic vest

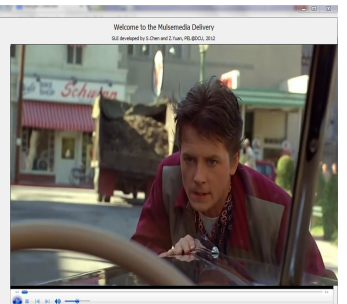

(d) Display interface

Fig. 2: Equipment and software used in the mulsemedia movie system

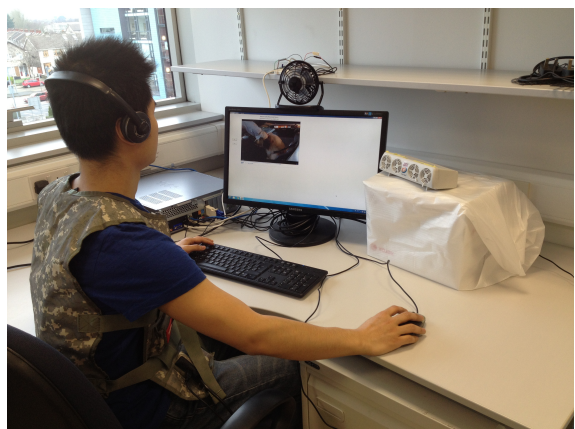

Fig. 3: Mulsemedia movie delivery test-bed

up to four types of scents at the same time. The on/off of each fan is controlled via a visual basic program. The vibration vest is available from TN Games who provides fully control (via a c++ program) of the haptic effect in terms of intensity, types, and duration. Figure 2 (d) presents the software interface which controls the on and off the three hardware devices and plays $\mathrm{A}$ the movie sequences for users.

\section{SUBJECTIVE TEST AND RESULTS ANALYSIS}

This section introduces the subjective test conducted and the results discovered.

\section{A. Subjective Test Description}

The subjective test was conducted in a separate lab room at Dublin City University (Ireland) without disturbing. Snapshot of the developed mulsemedia test-bed is shown in Figure 3. A user wears the vibration vest sitting in front of the screen. The USB fan and the olfaction dispenser are placed around $30 \mathrm{~cm}$ far away from the user. The windows of the room is closed during the test of one user and opened for a while in order to clear the olfaction lingered in the air. Testing
TABLE II: Mulsemedia movie sequences specification

\begin{tabular}{|c|c|c|c|c|}
\hline Movie & Quality & Framerate & Resolution & Bitrate \\
\hline \multirow{2}{*}{ "Jurassic Park" } & High & $30 \mathrm{fps}$ & $1280 \times 720$ & $2500 \mathrm{Kbps}$ \\
& Average & $24 \mathrm{fps}$ & $853 \times 480$ & $1100 \mathrm{Kbps}$ \\
\hline \multirow{2}{*}{ "Back To The Future" } & High & $30 \mathrm{fps}$ & $1280 \times 720$ & $2500 \mathrm{Kbps}$ \\
& Average & $24 \mathrm{fps}$ & $853 \times 480$ & $1100 \mathrm{Kbps}$ \\
\hline
\end{tabular}

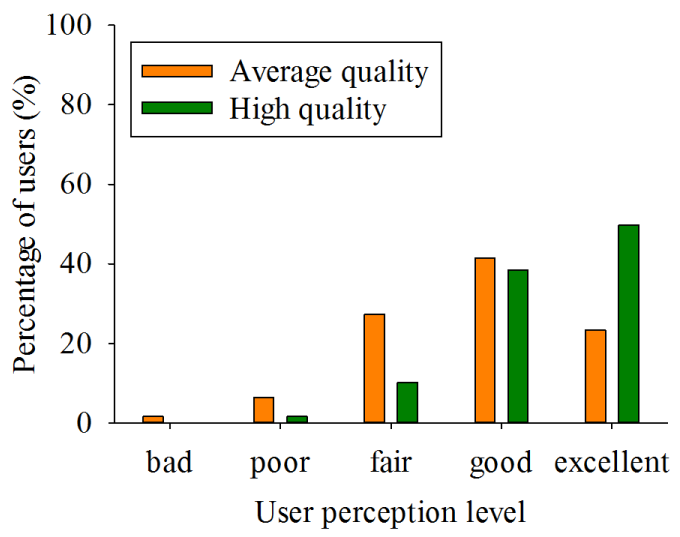

Fig. 4: User perception of the multimedia

environment was suggested according to ITU-T R.P.910 [12] and ITU-T R.P.911 [13]. Users were invited to complete the test and 128 copies of questionnaires were received. These users are from various backgrounds, e.g. students, engineers, scientists, etc., with an average age of 26. All of the users were asked to complete a pre-test to get familiar with the testing process. A questionnaire form [14] was designed to be finished by each user while watching the movie sequences. The questionnaire includes questions as follows: 1) the mulsemedia effects enhance the video content; 2) the mulsemedia effects are annoying; 3) the mulsemedia effects improve the sense of reality when watching the video; 4) the mulsemedia effects are distracting; 5) I enjoyed the experience. Each user was given six options to response the five questions above such as strongly disagree, slightly disagree, slightly agree, agree, strongly agree, and not notice. Each user watches 16 unique multimedia sequences taken from the movies Jurassic Park and Back To The Future. Each multimedia sequence was 30 seconds long. It took around 20 minutes to complete the subjective test for each user. In order to comparatively evaluate the effectiveness of the mulsemedia movie, users also watched traditional multimedia content which does not integrate any sensorial effects. User perception on both mulsemedia movie and traditional multimedia content are then compared as shown in the followed section.

\section{B. Results Analysis}

This section analyzes the questionnaires completed from the subjective test users. Figure 4 shows the user perception of traditional multimedia content which does not include any human sensorial effects. Users rate their perception levels of the multimedia content using terms bad, poor, fair, good, 
TABLE I: Sensorial Effect Descriptions attached to the Multimedia Content from "Jurassic Park" and "Back to the Future"

\begin{tabular}{|c|c|c|c|c|c|c|c|}
\hline \multirow{2}{*}{ Movie } & \multirow{2}{*}{ Sensorial Effect } & \multicolumn{3}{|c|}{ High Motion } & \multicolumn{3}{|c|}{ Low Motion } \\
\hline & & Clip & Scenario & Olfaction aroma & Clip & Scenario & Olfaction aroma \\
\hline \multirow{8}{*}{ "Jurassic Park" } & None & 1 & animal attack & - & 9 & animal attack & - \\
\hline & Haptic & 2 & animal attack & - & 10 & animal attack & - \\
\hline & Air & 3 & wind as car passing & - & 11 & subway coming & - \\
\hline & Olfaction & 4 & tear gas & burnt & 12 & decomposed anima & rubbish \\
\hline & Haptic,Air & 5 & vehicle moving and wind & - & 13 & pulled by parasail;wind & - \\
\hline & Haptic,Olfaction & 6 & animal attack and smoking & burnt & 14 & air crash;methane & methane \\
\hline & Air,Olfaction & 7 & wind and fire & burnt & 15 & ocean wind;wine & rock pools, wine \\
\hline & Haptic,Air,Olfaction & 8 & vehicle moving;wind, & forest & 16 & movement;gas;wind & methane \\
\hline \multirow{8}{*}{ "Back To The Future" } & None & 1 & animal attack & - & 9 & animal attack & - \\
\hline & Haptic & 2 & car crash & - & 10 & car crash & - \\
\hline & Air & 3 & wind & - & 11 & wind & - \\
\hline & Olfaction & 4 & smoke & burnt & 12 & burning bread & burnt \\
\hline & Haptic,Air & 5 & crash;wind & - & 13 & falling down; wind & - \\
\hline & Haptic,Olfaction & 6 & crash;manure & rubbish & 14 & sound waves;smoke & burnt \\
\hline & Air,Olfaction & 7 & wind;smoke & burnt & 15 & smoke;wind & burnt \\
\hline & Haptic,Air,Olfaction & 8 & car moving;wind;smoke & burnt & 16 & car moving;fire;wind & methane \\
\hline
\end{tabular}

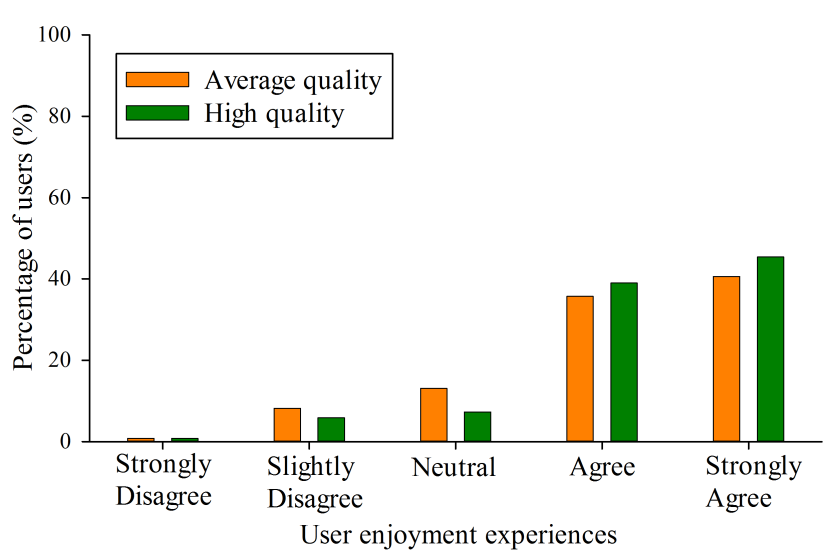

Fig. 5: User enjoyment of the mulsemedia

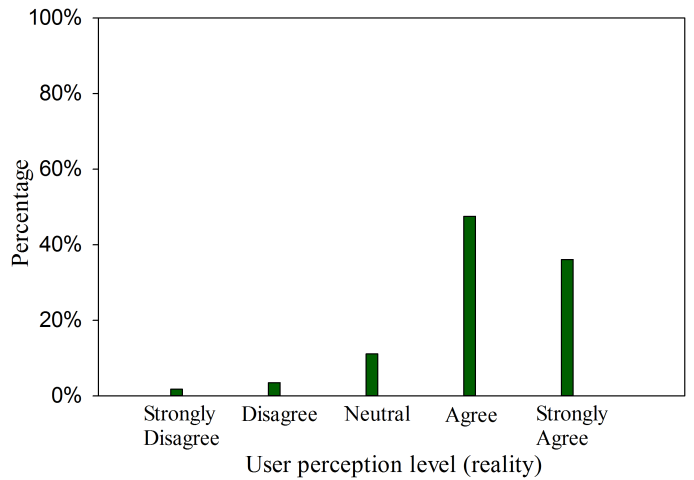

Fig. 6: The mulsemedia sequence enchances the sense of reality

and excellent. It is shown that the large majority of users rates average and high quality multimedia sequences good $(41.4 \% / 23.4 \%)$ and excellent (38.5\%/49.7\%), respectively. Figure 5 shows the user enjoyment levels for the mulsemedia content. The results demonstrate that the majority of users (76.3\%/74.4\%) agree that regardless of the video quality level,

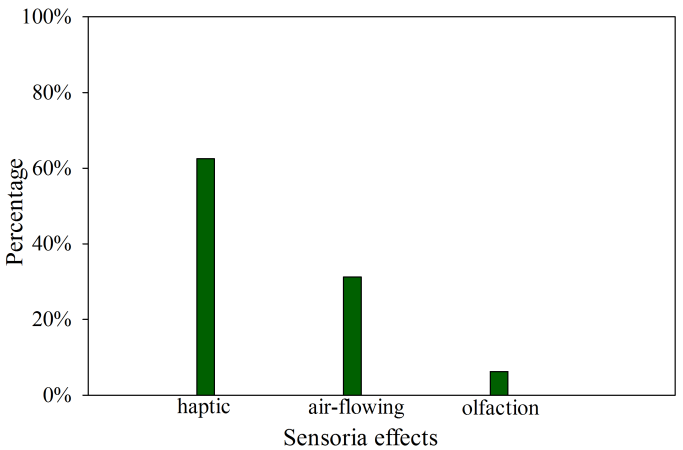

Fig. 7: The overall preference levels of different sensorial effects

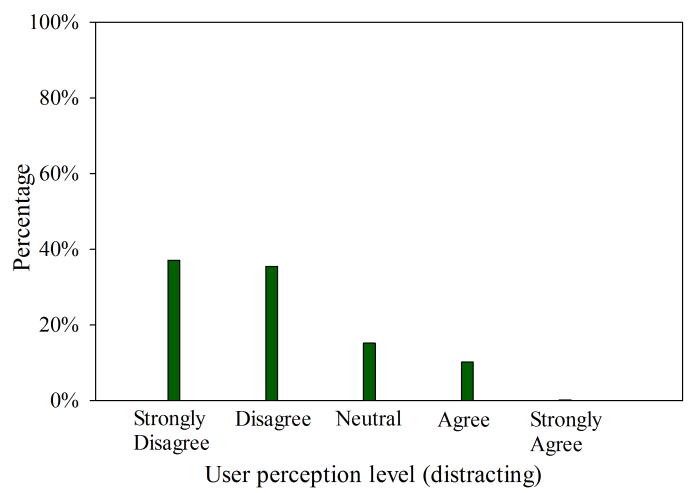

Fig. 8: The mulsemedia sequence is distracting

mulsemedia content increases user enjoyment.

Users are asked to answer a questionnaire statement, "The sensorial effects improve the sense of reality when watching the video" with one of the five response: "strongly disagree", "disagree", "netural", "agree", and "strongly agree". The results are presented in Figure 6, which shows that $47.5 \%$ of users agree and $36.1 \%$ of users strongly agree $(83.6 \%$ of users tend to agree) that the mulsemedia sequence ehances the sense of reality. Figure 7 shows the results of users' responses to 


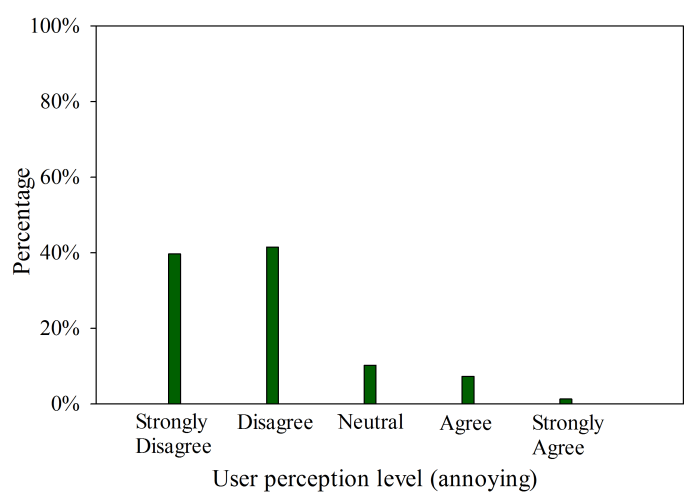

Fig. 9: The mulsemedia sequence is annoying

questionnaire statement "Which sensorial effect do you prefer (or you like the best)?". It is shown that $62.5 \%$ of users prefer haptic, $31.25 \%$ of users prefer air,and $6.25 \%$ of users prefer olfaction.

Figure 8 and Figure 9 present the results of users' responses to questionnaire stamentes "The sensorial effects are distracting" and "The sensorial effects are annoying.", respectively. The results show that: 1) $37.1 \%$ of users strongly disagree and $35.5 \%$ of users slightly disagree that the mulsemedia sequences are distracting; 2) $39.7 \%$ of users strongly disagree and $41.5 \%$ of users slightly disagree that the mulsmedia sequences are annoying.

Additionally, the following testing conclusions can be drawn.

1) Without mulsemedia effects as inputs, the large majority of users have noticed the differences in multimedia quality. However, when delivering mulsemedia content, there is no statistical difference between user enjoyment levels when exposed to average and high quality sequences, respectively. In additiaon, the user enjoyment levels were maintained high when lower multimedia quality sequences have been used in conjunction with multiple sensorial effects. This is as the multiple sensorial effects have partly masked the video quality decrease.

2) Among the three sensorial effects, haptic, olfaction, and air-flowing, haptic is the most popular effect. The second preferable effect is air-flowing.

3) By analysing the questionnaires it can be noted that synchronization between sensorial effects and multimedia content needs to be precise, especially when olfaction effect is included. This is because the scent lingers in the air.

\section{CONCLUSION}

This paper presents a novel mulsemedia movie delivery system which integrates multiple human sensorial media types (i.e. haptic, olfaction, and air-flowing) into traditional multimedia sequences. Investigation on user perceptions of the mulsemedia movie is conducted via subjective test. The testing results indicate that the integration of human sensorial media can partly mask the video quality decrease. Additionally, haptic is the favorite sensorial effect for most users. In the case of olfaction, unpleasant scent should be avoided as it tends to decrease user perceptions. Future works will focus on the effects of mulsemedia input synchronization and dynamic adaptation of the multi-sensorial movie sequence to variable network conditions and user requirement.

\section{REFERENCES}

[1] K. Acuna, "3 Signs That 3D Movies Are The Way Of The Future", Business Insider, Jan. 2013. http://www.businessinsider.com/3d-movieshave-a-future-in-hollywood-2013-1.

[2] J. G. Apostolopoulos, P. A. Chou, B. Culbertson, T. Kalker, M. D. Trott, and S. Wee, The Road to Immersive Communication, in Proc. IEEE, no.4, vol.200, pp.974 990, 2012.

[3] G. Ghinea, S.R. Gulliver and F. Andres (eds.), Multiple Sensorial Media Advances and Applications: New Developments in MulSeMedia, IGI Global, 2011

[4] Z. Yuan and G.-M. Muntean, "A Prioritized Adaptive Scheme for Multimedia Services over IEEE 802.11 WLANs", IEEE Trans. Network and Service Management, (DOI) 10.1109/TNSM.2013.110513.130490, Nov.2013.

[5] Z. Yuan, H. Venkataraman, and G.-M. Muntean,'iPAS:An User Perceived Quality-based Intelligent Prioritized Adaptive Scheme for IPTV in Wireless Home Networks", IEEE International Symposium on Broadband Multimedia Systems and Broadcasting (BMSB), Shanghai, China, Mar. 2010, pp.1-6.

[6] G.-M. Muntean, N. Cranley, Resource Efficient Quality-Oriented Wireless Broadcasting of Adaptive Multimedia Content, IEEE Trans. on Broadcasting, vol. 53, no. 1, part 2, March 2007, pp. 362-368.

[7] J.N. Kaye, Making Scents: Aromatic Output for HCI, Interactions, vol.11, no.1, pp.48 61, 2004.

[8] P. Kortum, HCI beyond the GUI: Design for haptic, speech, olfactory, and other nontraditional interfaces. Burlington, MA: Morgan Kaufmann, 2008.

[9] E. Richard, A. Tijou, P. Richard and J.-L. Ferrier, Multi-modal virtual environments for education with haptic and olfactory feedback, Virtual Reality, no.3-4, vol.10, pp.207-225, 2006.

[10] J. Ryu, and G.J. Kim, Using a vibro-tactile display for enhanced collision perception and presence, in Proc. ACM symposium on Virtual reality software and technology, Hong Kong, China, Nov.2004, pp.89-96.

[11] K. Kahol, P.Tripathi, T. Mcdaniel, L. Bratton, and S. Panchanathan, Modeling context in haptic perception, rendering, and visualization, ACM Trans. Multimedia Computing, Communications and Applications, vol.2, no.3, pp.219-240, 2006.

[12] ITU-T Recommendation P.910, Subjective Video Quality Assessment Methods for Multimedia Applications, Apr. 2008.

[13] ITU-T Recommendation P.911, Subjective Audiovisual Quality Assessment Methods for Multimedia Applications, Dec. 1998.

[14] Z. Yuan, G. Ghinea and G.-M. Muntean, Documents, Technical Report, Performance Engineering Lab, Dublin City University, Ireland, Dec.2013, [Online]. Available: http://elm.eeng.dcu.ie/ yuanzh/papers/appendix.pdf. 